\title{
Dynamic computed tomography to determine cardiac output in patients with left ventricular assist devices
}

Subha V. Raman, MD, MSEE, Tam Tran, BS, Orlando P. Simonetti, PhD, and Benjamin Sun, MD

Objective: The purpose of this study was to develop and test a novel, noninvasive means of estimating cardiac output in patients with continuous-flow left ventricular assist devices.

\begin{abstract}
Methods: Based on the indicator dilution principle, we estimated cardiac output from signal intensity versus time recordings during first-pass imaging with contrast-enhanced computed tomography. To account for recirculation, a gamma variate function was modified to model the indicator concentration curve. A proportionality constant for 64-slice computed tomography data acquisition was derived relating computed tomography Hounsfield units to indicator concentration. Validity of the equation was tested in ambulatory patients with continuous-flow left ventricular assist devices who underwent simultaneous computed tomography and Swan-Ganz catheter thermodilution-based measurement of cardiac output at various left ventricular assist device rpm settings. Agreement between measurements was testing using kappa statistic.
\end{abstract}

Results: Pairwise comparison of calculated output using the dynamic computed tomography test bolus technique versus thermodilution output measurements yielded good agreement $(P=.03)$. The output calculated using dynamic computed tomography underestimated the thermodilution output measurement by $0.54 \pm 0.37 \mathrm{~L} / \mathrm{min}$ (95\% confidence interval, 0.66-0.94).

Conclusions: Noninvasive estimation of cardiac output from left ventricular assist devices is feasible using firstpass dynamic computed tomography. By affording simultaneous good visualization of the device in situ, computed tomography may be useful for noninvasive assessment of location and function of ambulatory patients with left ventricular assist devices.

Each year, heart failure results in 250,000 deaths in the United States, and limited availability of donor hearts makes cardiac transplantation a viable option for only a fraction of end-stage heart failure patients. In this setting, ventricular assist devices (VADs) provide a bridge to transplantation or act simply as destination therapy without subsequent transplantation. ${ }^{1-3}$ The typical VAD configuration for left heart support places (1) the rotor device in the left peritoneal cavity, (2) an input cannula in the left ventricular apex, and (3) an output cannula sewn into the ascending aorta. By either pulsatile or continuous flow, VADs generate the cardiac output needed for end-organ perfusion.

Cardiac output may rely entirely on the VAD in the setting of end-stage heart failure or may be a combination of residual native heart function and VAD output. The internal flow estimator of axial flow (one of the continuous flow) LVADs is neither consistently nor reliably accurate. A noninvasive assessment of cardiac output could help guide adjustment and potential weaning of VAD support in patients, particu-

\footnotetext{
From The Ohio State University, Columbus, Ohio.

This work was supported by a NIH grant No. R21 HL080474-01.

Dr Sun reports consulting and lecture fees from Thoratec and equity ownership and lecture fees from Abiomed. Drs Simonetti and Raman report grant support from Siemens.

Received for publication July 30, 2008; revisions received Oct 1, 2008; accepted for publication Oct 9, 2008.

Address for reprints: Subha V. Raman, MD, MSEE, The Ohio State University Davis Heart and Lung Research Institute, 473 West 12th Ave, Suite 200, Columbus, OH 43210 (E-mail: raman.1@osu.edu).

J Thorac Cardiovasc Surg 2009;137:1213-7

$0022-5223 / \$ 36.00$

Copyright (C) 2009 by The American Association for Thoracic Surgery doi:10.1016/j.jtcvs.2008.10.043
}

larly those with recovering left ventricular function. Metal artifact may limit ultrasound-based measurements, and multiple device components preclude interrogation with magnetic resonance. Mahnken and colleagues ${ }^{4,5}$ have shown that dynamic computed tomography (CT) could provide accurate assessment of cardiac output using the concept of indicator dilution applied to noninvasive tracking of iodinated contrast. We sought to extend this work to measure cardiac output in response to variable VAD output.

\section{METHODS}

\section{Cardiac Output Model}

Cardiac output can be calculated from the time enhancement curve based on the Stewart-Hamilton equation:

$C O=\frac{Q}{\int_{0}^{\infty} c(t) d t}$

where $C O$ is cardiac output and $Q$ is the amount of contrast used in milligrams. Using $20 \mathrm{~mL}$ of iodinated contrast agent with a density of $350 \mathrm{mg}$ / $\mathrm{mL}$ yielded a $Q$ value of $7000 \mathrm{mg}$ of iodine. The function $c(t)$ is the indicator concentration. To account for recirculation, a gamma variate function is used to model the indicator concentration curve, an excellent model of the first pass of fluids through the cardiovascular system. ${ }^{6}$ Mahnken and colleagues ${ }^{4}$ showed that the Stewart-Hamilton equation can be simplified to:

$C O=K * \frac{Q}{A}$,

where $A$ is the area under the indicator concentration curve and $K$ is a proportionality constant that converts $c(t)$ from units of signal intensity (Hounsfield units) to units of concentration. The $K$ value must be adjusted based on the specific CT machine configuration and scan parameters. 


\section{Abbreviations and Acronyms \\ $\mathrm{CT}=$ computed tomography \\ $\mathrm{VAD}=$ ventricular assist device}

\section{Determination of $\mathbf{K}$ Value}

The $C O=K * \frac{Q}{A}$ equation can be arranged into $K=\frac{C O * A}{O}$. The $Q$ value is known, and $C O$ can be computed as left ventricular stroke volume $\times$ heart rate. The stroke volume is measured from contiguous short-axis cine images covering the left ventricle by tracing end-systolic and enddiastolic areas in each slice. Knowing the thickness of each slice, the enddiastolic volume and end-systolic volume are computed using Simpson's rule: Stroke volume $=$ end-diastolic volume - end-systolic volume. $A$ is calculated by integration of the dynamic contrast enhancement signal acquired in the ascending aorta. The data necessary to estimate $K$ are routinely obtained in patients undergoing coronary CT angiography using a test bolus for contrast timing. Images from test bolus injections obtained in 23 patients undergoing coronary CT angiography were used to determine the $K$ value using the formula above; the average $K$ value over these patients was 13.6.

\section{In vivo Validation Studies}

Patients in heart failure with continuous-flow left heart VADs scheduled for right heart catheterization were prospectively enrolled to undergo simultaneous right heart catheterization and dynamic CT. All patients provided written informed consent to participate in this Institutional Review Board-approved human subjects investigation. Right heart catheterization was performed in all cases to quantify pulmonary hypertension and invasive hemodynamics as part of pre-heart transplantation planning. Excluded from enrollment were patients with allergy to iodinated contrast agent or significant renal insufficiency (glomerular filtration rate $<30 \mathrm{~mL} \cdot \mathrm{min}^{-1}$. $\mathrm{m}^{-2}$ ). No patient had active thyroid disease or childbearing potential.

\section{CT Protocol}

Upon completion of cardiac catheterization, the fluid-filled end-hole catheter was secured with the tip in a proximal branch pulmonary artery (confirmed by fluoroscopy), and patients were transported in the supine position to the CT scanner (Somatom 64, Siemens Medical Solutions, Inc, Forchheim, Germany). After scout images were obtained, a single axial slice location in the ascending aorta distal to the anastomosis of the output cannula was selected (Figure 1). The protocol for cardiac output measurement at different VAD settings was then as follows: first, an invasive cardiac output measurement was obtained using thermodilution technique. Next, a 20 $\mathrm{mL}$ bolus of isosmolar contrast agent (Visipaque 350 GE Healthcare, Waukesha, Wis) was injected followed by $20 \mathrm{~mL}$ of saline, both via peripheral vein at a rate of $4 \mathrm{~mL} / \mathrm{s}$. During the injection, repeated single-slice imaging was performed at the predetermined axial location with a temporal resolution of $750 \mathrm{~ms}$, resulting in 40 to 50 images. This test bolus scan was obtained using minimal radiation dose of $2 \mathrm{mSv}$. The VAD output (rpm) was then increased or decreased, and after an equilibration period of 8 to 10 minutes, another test bolus scan was obtained. For each patient, a total of $5 \mathrm{VAD}$ output settings were queried in this fashion.

\section{Image Analysis}

The test bolus scan yields 40 images at the same axial location over time (Figure 2), allowing a region of interest to be drawn in the distal ascending aorta to derive a signal-intensity versus time curve. The data points were exported as a text file allowing transfer to a PC for computation using the formula derived above (MATLAB software, The Mathworks, Inc, Natick, Mass).
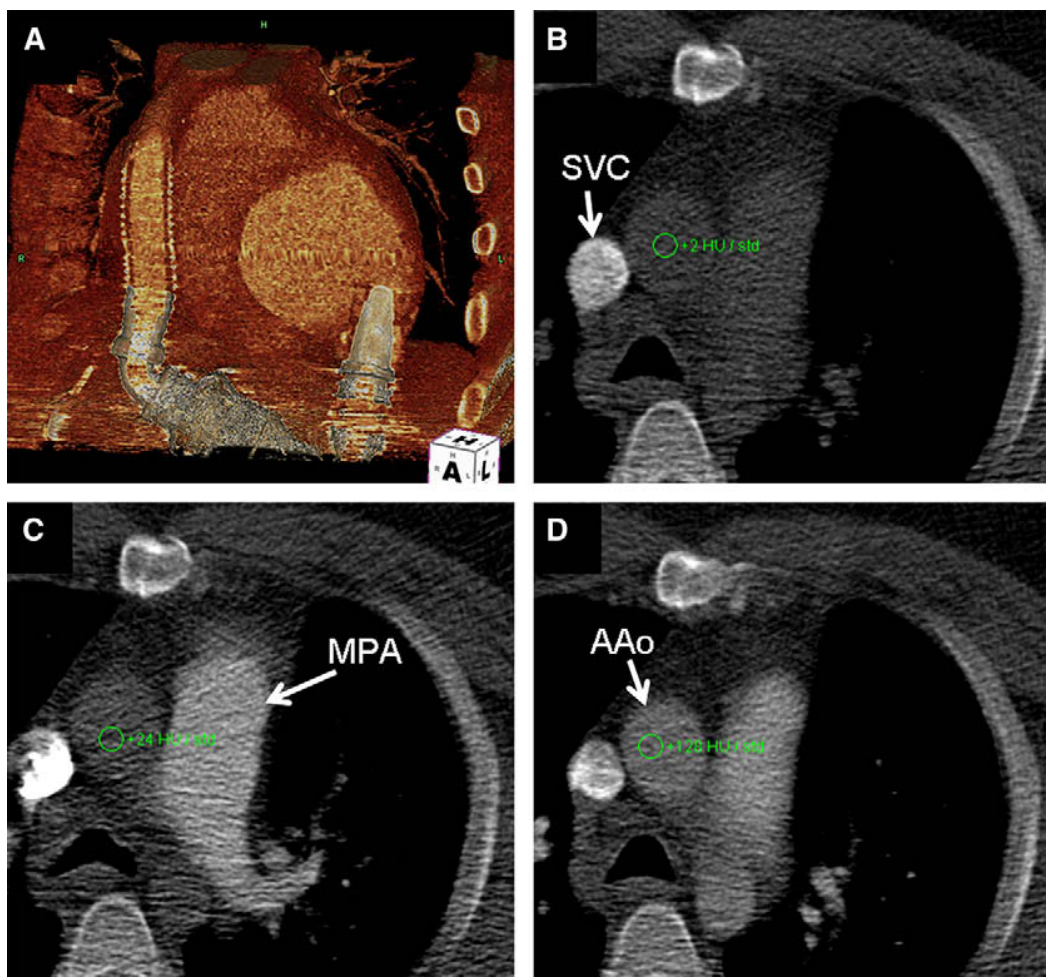

FIGURE 1. A, Volume rendering of computed tomography (CT) images demonstrates the position of the outflow cannula and aorta in a patient with a left ventricular assist device. B, C, and D are axial CT images of the test bolus scan at serial time points after injection of contrast showing subsequent enhancement of the superior vena cava $(S V C)$, main pulmonary artery $(M P A)$, and ascending aorta $(A A o)$. The average signal intensity within a region of interest placed in the AAo is 2, 24, and 128 Hounsfield units on these serial images. 


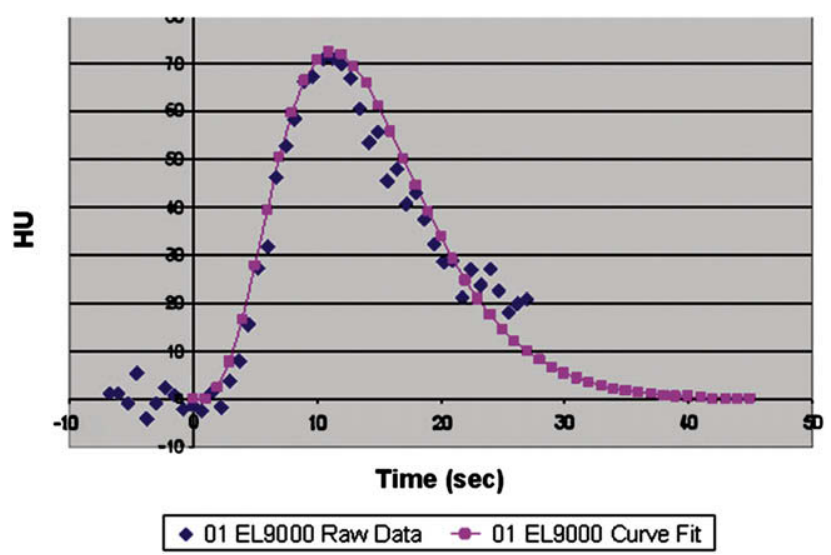

FIGURE 2. The average signal intensity in a region of interest drawn on the test bolus images in the distal ascending aorta versus time during injection of a small bolus of contrast. The blue dots represent the raw data, and the dotted pink line represents the gamma variate curve fit. $H U$, Hounsfield units.

\section{Statistical Analysis}

Comparison of calculated output using the dynamic CT test bolus method to thermodilution output measured by invasive right heart catheterization was performed using Wilcoxon signed-rank test of equality of pairs. Evaluation of systematic bias between noninvasive and invasive measurements was performed using Bland-Altman analysis. ${ }^{7,8}$

\section{RESULTS}

All 4 patients successfully completed the multiple test bolus dynamic CT acquisition protocol. Image quality was adequate for analysis in all acquisitions.

Table 1 summarizes the data collected from 4 patients and multiple VAD rpm settings for each patient. Pairwise comparison of calculated output using the dynamic CT test bolus technique versus thermodilution output measurements yielded good agreement (Figure 3, $A ; P=.03$ ). For $10 \mathrm{VAD}$ settings in the 4 patients, the readout of estimated cardiac output on the device readout was recorded. This correlated very poorly with invasive measurements (Figure $3, B$ ).

The output calculated using dynamic CT tended to underestimate the thermodilution output measurement by $0.54 \pm$ $0.37 \mathrm{~L} / \mathrm{min}$ (95\% confidence interval 0.66-0.94; Figure 4). The 2 points that fell outside of the standard Bland-Altman boundaries ( \pm 1.96 standard deviation) involved 1 VAD setting at $7200 \mathrm{rpm}$ in a patient whose usual setting was 9000 rpm and another at a VAD setting of 9200 compared to usual setting of 8200 with concomitant nonsustained ventricular tachycardia during data acquisition.

\section{DISCUSSION}

We adapted the indicator dilution technique for noninvasive estimation of cardiac output and showed good agreement with invasive measurements in patients with continuous-flow left VADs. By tracking a contrast bolus over time as previously developed for native heart cardiac output, we have demonstrated feasibility of noninvasive cardiac output measurement in patients with VADs.

This work highlighted several important aspects of left VAD physiology. First, we acquired our CT measurements in the ascending aorta distal to the outflow cannula insertion site. The measured cardiac output, therefore, would reflect the combination of left VAD output as well as any native cardiac output. We used this location because it is this output that is most relevant for end-organ perfusion, the ultimate

TABLE 1. Ventricular assist device settings and hemodynamic measurements

\begin{tabular}{|c|c|c|c|c|c|}
\hline Patient No. & RPM setting & $\begin{array}{c}\text { Dynamic CT } \\
\text { CO }(\mathrm{L} / \mathrm{min})\end{array}$ & $\begin{array}{l}\text { Dynamic CT CI } \\
\left(\mathbf{L} \cdot \min ^{-1} \cdot \mathbf{m}^{-2}\right)\end{array}$ & $\begin{array}{c}\text { Thermodilution } \\
\text { CO (L/min) }\end{array}$ & $\begin{array}{c}\text { Thermodilution } \\
\text { CI }\left(\mathbf{L} \cdot \mathbf{m i n}^{-1} \cdot \mathbf{m}^{-2}\right)\end{array}$ \\
\hline 1 & 10000 & 3.4 & 1.5 & 4.3 & 1.9 \\
\hline 1 & 10400 & 3.8 & 1.6 & 4.6 & 2.0 \\
\hline 1 & 10800 & 5.1 & 2.2 & 5.0 & 2.2 \\
\hline 1 & 9000 & 3.7 & 1.6 & 4.0 & 1.7 \\
\hline 2 & 9400 & 3.1 & 1.7 & 3.3 & 1.8 \\
\hline 2 & 8000 & 3.7 & 2.0 & 4.0 & 2.2 \\
\hline 2 & 7000 & 3.2 & 1.8 & 3.9 & 2.2 \\
\hline 2 & 8000 & 3.0 & 1.7 & 3.3 & 1.8 \\
\hline 2 & 9400 & 3.9 & 2.2 & 4.6 & 2.5 \\
\hline 3 & 8200 & 4.0 & 2.4 & 4.5 & 2.7 \\
\hline 3 & 9200 & 5.0 & 3.0 & 4.4 & 2.7 \\
\hline 3 & 10400 & 3.8 & 2.3 & 4.4 & 2.7 \\
\hline 3 & 8200 & 4.2 & 2.6 & 5.0 & 3.0 \\
\hline 3 & 7200 & 2.9 & 1.8 & 4.4 & 2.7 \\
\hline 4 & 9000 & 5.3 & 2.3 & 6.3 & 2.8 \\
\hline 4 & 8000 & 4.6 & 2.0 & 4.7 & 2.1 \\
\hline 4 & 9000 & 5.2 & 2.3 & 5.6 & 2.5 \\
\hline 4 & 10000 & 5.4 & 2.4 & 6.3 & 2.8 \\
\hline 4 & 9000 & 5.2 & 2.3 & 6.0 & 2.7 \\
\hline
\end{tabular}

$\mathrm{CO}$, Cardiac output; $\mathrm{CI}$, cardiac index. 

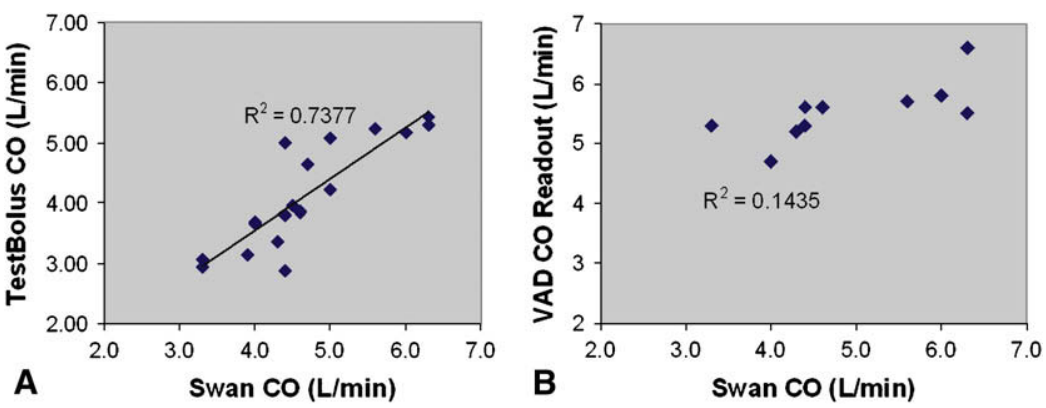

FIGURE 3. A, Linear regression between calculated cardiac output using the dynamic computed tomography technique and measured cardiac output using catheter thermodilution shows good agreement, with correlation coefficient of 0.74 . B, Linear regression between the device cardiac output readout and thermodilution measurement shows poor agreement, with correlation coefficient of 0.14 .

goal of VAD therapy. The acquisition plane could have been placed more proximally such that both the outflow cannula and aortic root proximal to the outflow cannula could be seen in cross section, allowing generation of individual signal intensity-time curves for device and native heart. In this manner, our noninvasive technique could be applied to further study the relative contributions of left VAD function and native heart function in various states of heart failure and recovery. Second, we studied patients with continuous-flow devices rather than pulsatile devices. The relative physiologic merits of one output mechanism versus the other continue to be debated, though most current devices tend to use a continuous-flow model. Quantitative comparisons, particularly using preclinical models, could be performed serially using our technique. Third, the response to increases and decreases in VAD rpm settings are nonlinear and also afford insights into the optimal VAD settings in a given patient under various hemodynamic circumstances.

Echocardiography is the most widely used modality for assessment of patients post-VAD placement. ${ }^{9,10}$ A host of secondary findings, ranging from closure of the aortic valve

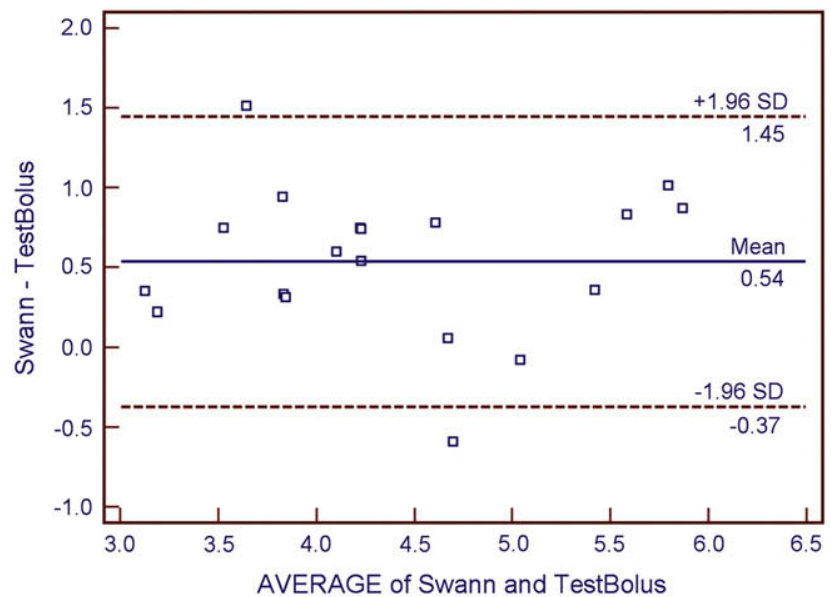

FIGURE 4. Bland-Altman plot shows the difference between the two cardiac output $(\mathrm{CO})$ measurement techniques versus the average value obtained with the 2 techniques. The mean difference was $0.54 \mathrm{~L} / \mathrm{min}$, with a $95 \%$ confidence interval difference of 0.66 to $0.94 \mathrm{~L} / \mathrm{min}$. throughout the cardiac cycle to absence of significant mitral regurgitation, allow echo-based imaging to infer adequate decompression of the left ventricle and appropriate VAD functioning. However, severe acoustic shadowing may limit visualization of cannula placement. CT overcomes these limitations, and with the additional hemodynamic data that may be provided based on the results of our work, this modality may offer additional information regarding VAD location and performance not readily obtained with echocardiography alone.

When performing procedures involving ionizing radiation, dose should be minimized given uncertainty as to long-term health effects at the albeit small radiation doses used for diagnostic imaging. The minimal dose required for the single timing bolus CT scan for cardiac output measurement as described in this work pales in comparison to the doses patients with VADs are typically exposed to over the course of multiple invasive coronary angiograms, nuclear viability studies, endomyocardial biopsy procedures, and other sources of radiation. Furthermore, the theoretical lifetime risks must be weighed against the short-term health care needs in this population, whose survival beyond 1 year may depend heavily on appropriate assist device functioning. Additionally, the noninvasive volumetric visualization of the device in situ afforded by $\mathrm{CT}$ is unique compared with other diagnostic modalities and may be useful in cannulae localization or in defining other positional contributors to VAD output.

Examination of the contribution of backflow in the ascending aorta was not performed, as this component is likely included in the net aortic flow measurement obtained with our technique. This nongated acquisition over approximately 40 seconds includes multiple cardiac cycles, precluding assessment of individual phasic changes in output. The type of VAD and cannulation sites may also impact the results obtained with this technique and warrant further study. Peripheral venous access has been cited as a potential limitation of this technique.

The accuracy of the flow estimator on the HeartMate II (Transonics Systems, Inc, Ithaca, NY) is quite variable, 
motivating this work to obtain more accurate measures of flow device-estimated cardiac output. Improved techniques for serial flow assessment may help guide studies of heart unloading and device output optimization.

In summary, we have shown that dynamic CT may provide accurate noninvasive estimates of cardiac output in patients with continuous-flow left VADs. This approach may be useful in addressing clinical questions regarding VAD function as well as investigations regarding VAD physiology.

We thank the OSU Medical Center's Clinical Engineering staff for their support of this work. The cooperation of the OSU Cardiac Transplant program, particularly Ms. Sherri Weissman, is also appreciated.

\section{References}

1. Camp D. The left ventricular assist device (LVAD). A bridge to heart transplantation. Crit Care Nurs Clin North Am. 2000;12:61-8.
2. DeRose JJ Jr, Umana JP, Argenziano M, Catanese KA, Gardocki MT, Flannery M, et al. Implantable left ventricular assist devices provide an excellent outpatient bridge to transplantation and recovery. J Am Coll Cardiol. 1997;30: 1773-7.

3. Goldstein DJ, Oz MC, Rose EA. Implantable left ventricular assist devices. N Engl J Med. 1998;339:1522-33.

4. Mahnken AH, Klotz E, Hennemuth A, Jung B, Koos R, Wildberger JE, Gunther RW. Measurement of cardiac output from a test-bolus injection in multislice computed tomography. Eur Radiol. 2003;13:2498-504.

5. Mahnken AH, Rauscher A, Klotz E, Muhlenbruch G, Das M, Gunther RW, Wildberger JE. Quantitative prediction of contrast enhancement from test bolus data in cardiac MSCT. Eur Radiol. 2007;17:1310-9.

6. Thompson HKJ, Starmer FC, Whalen RE, McIntosh HD. Indicator transit time considered as gamma variate. Circ Res. 1964;14:502-15.

7. Bland J, Altman D. Statistical method for assessing agreement between two methods of clinical measurement. Lancet. 1986;i:307-10.

8. Bland J, Altman D. Measuring agreement in method comparison studies. Stat Methods Med Res. 1999;8:135-60.

9. Catena E, Milazzo F. Echocardiography and cardiac assist devices. Minerva Cardioangiol. 2007;55:247-65.

10. Chumnanvej S, Wood MJ, MacGillivray TE, Melo MF. Perioperative echocardiographic examination for ventricular assist device implantation. Anesth Analg. 2007; 105:583-601 